\title{
Lower FRAX scores but similar femoral neck aBMD in UK dwelling postmenopausal South Asian women as compared with same age Caucasian women
}

\author{
A.L. Darling, K.H. Hart, S.A. Lanham-New
}

\section{Department of Nutritional Sciences, School of Biosciences and Medicine, Faculty of Health and Medical Sciences, University of Surrey, Guildford GU2 7XH, UK.}

\section{INTRODUCTION}

- It is unclear whether western dwelling South Asian (SA) postmenopausal women have a different fracture risk to that of the native Caucasian $(C)$ population.

- A recent U.S. study found that South Asian women had a higher prevalence of femoral neck osteoporosis than Caucasian women and a higher incidence of wrist fracture than Chinese women, although they did not differ from White Caucasian women on these measures (Khandewal et al. 2012; Lofthus et al. 2008).

- Moreover, the WHO Fracture Risk Assessment Tool (FRAX) has not been used previously to compare predicted risk of fractures in western dwelling South Asian women with same-age Caucasian women.

\section{AIMS}

- The aim of this work was to compare FRAX scores and Femoral Neck areal BMD (FNaBMD) between UK dwelling Caucasian and South Asian women.

\section{METHODS}

- This analysis used data from $n=35 S A$ and $n=136 \quad C$ postmenopausal women (mean [SD] age $=59$ [6] years and 61 [5] years respectively) in the D-FINES I study (UK, 2006-2007).

- FRAX score was calculated using FN aBMD and background demographic data, via the online tools at www.shef.ac.uk/FRAX/.

- The SA women were scored on both the India and UK tools as it was unclear which was likely to be the best epidemiological fit.

\section{FUNDING AND DISCLOSURES}

- The D-FINES I study was funded by the UK Food Standards Agency (Project N05064). All views expressed as those of the authors alone and do not constitute government advice.

- ALD AND KHH have no conflict of interest to disclose. SAL-N discloses that she is project director of D3-TEX Ltd

\section{RESULTS}

- There was no difference between the two ethnic groups for FN aBMD $(\mathrm{P}=0.44)$. However, the 10 year fracture risk (\%) was lower in the SA group (using the India tool) than the $\mathrm{C}$ group by $50-60 \%$ (see table 1,Figure 1 A). As seen in Figure 1B, women's understanding of their own family history of hip fracture had an influence on the results, with an attenuated ethnic difference in fracture risk when those who were unsure were excluded.
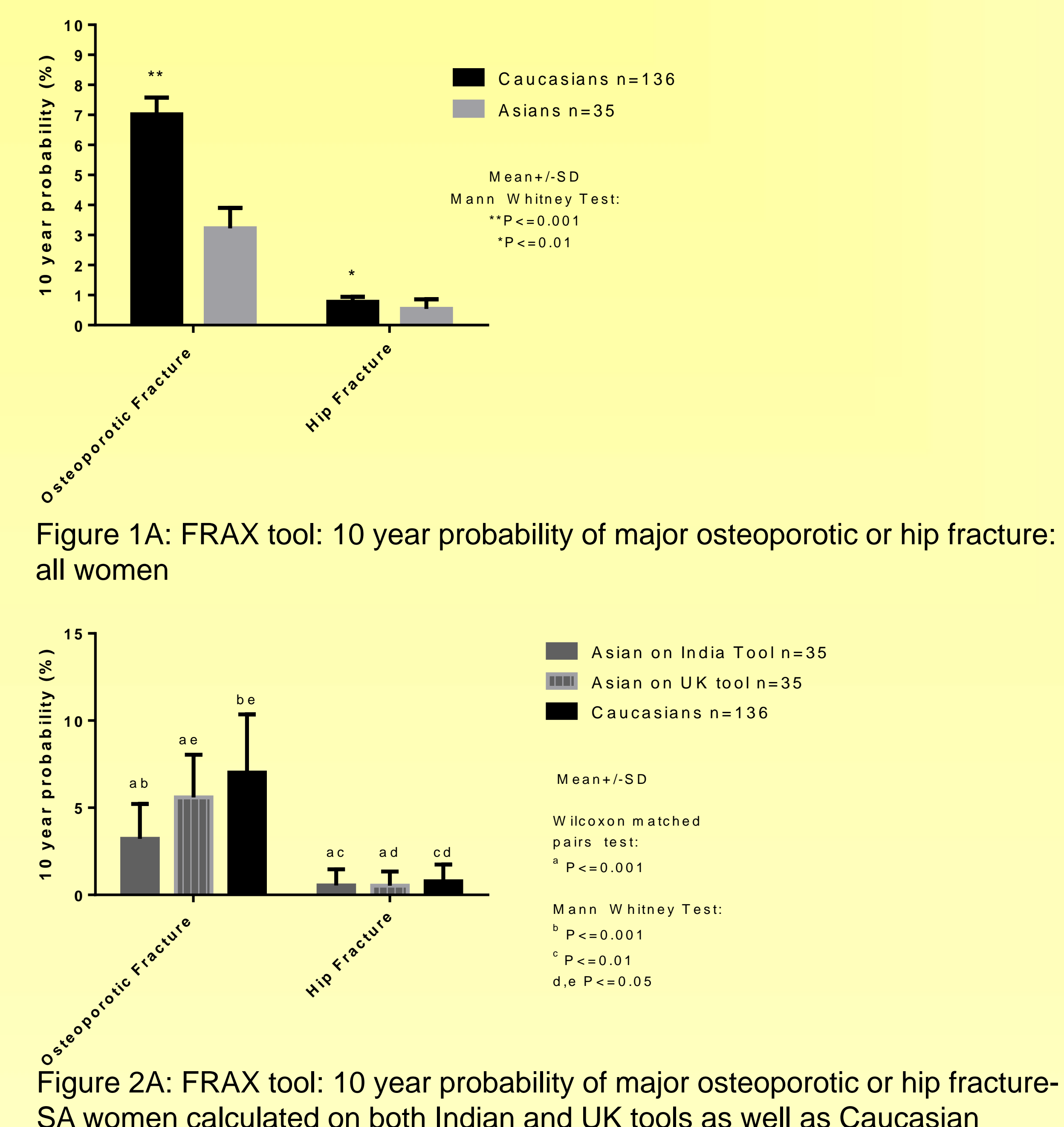

SA women calculated on both Indian and UK tools as well as Caucasian comparison group: all women
Table 1: FRAX score and arial BMD by ethnic group

\begin{tabular}{|c|c|c|c|c|c|c|c|}
\hline Group (Tool) n & \multicolumn{2}{|c|}{$C(U K) n=136$} & \multicolumn{3}{|c|}{ SA (India) n=35 } & \multicolumn{2}{|c|}{ SA (UK) n=35 } \\
\hline $\begin{array}{l}10 \text { year } \\
\text { Probability: }\end{array}$ & Median & IQR & Median & IQR & $P^{*}$ & Median & IQR \\
\hline O. Fracture \% & 6.5 & 4.4 & 2.5 & 2.5 & $<0.001$ & $4.7^{¥}$ & 2.5 \\
\hline H. Fracture \% & 0.4 & 0.8 & 0.2 & 0.5 & 0.006 & $0.2^{¥}$ & 0.6 \\
\hline & Mean & SD & Mean & SD & $\mathrm{P} \neq$ & & \\
\hline $\begin{array}{l}\text { FN aBMD } \\
\left(\mathrm{g} / \mathrm{cm}^{2}\right)\end{array}$ & 0.76 & 0.11 & 0.77 & 0.10 & 0.44 & - & - \\
\hline $\begin{array}{l}\text { LS aBMD } \\
\left(\mathrm{g} / \mathrm{cm}^{2}\right)\end{array}$ & 0.94 & 0.16 & 0.92 & 0.14 & 0.12 & - & - \\
\hline
\end{tabular}

*Mann Whitney: C (UK) vs. SA (India), O.=Osteoporotic, H.=Hip,, \#ANCOVA P value for BMI adjusted data $¥$ Statistically different from SA (India) and C (UK) using Wilcoxon test $(P<0.005)$. FN= Femoral Neck, LS=Lumbar Spine

\section{CONCLUSION}

- The reduced FRAX score in the SA group has not been reported previously and may be due to a variety of lifestyle and medical factors, as well as possible differential reporting of family history of fracture. Further research is warranted with respect to future fracture risk in this ethnic group.

- These data will be helpful in the clinical setting by showing how South Asian fracture risk varies by tool used, as well as by whether South Asian women are able to confidently report family history of fracture or not. It may be the case that a FRAX tool specifically for UK dwelling South Asians is now required. 